\title{
A tale of two operations: re-excision as a quality measure
}

\author{
Leisha C. Elmore, Julie A. Margenthaler \\ Department of Surgery, Washington University School of Medicine, St. Louis, MO, USA \\ Correspondence to: Julie A. Margenthaler, MD, FACS. Department of Surgery, Washington University School of Medicine, 660 S. Euclid Ave., \\ Campus Box 8109, St. Louis, MO 63110, USA. Email: jmargenthaler@wustl.edu. \\ Provenance: This is an invited article commissioned by the Editorial Office of Gland Surgery. \\ Comment on: Landercasper J, Bennie B, Ahmad HF, et al. Opportunities to reduce reoperations and to improve inter-facility profiling after initial \\ breast-conserving surgery for cancer. A report from the NCDB. Eur J Surg Oncol 2019;45:2026-36.
}

Submitted Nov 12, 2019. Accepted for publication Nov 28, 2019.

doi: 10.21037 /gs.2019.11.24

View this article at: http://dx.doi.org/10.21037/gs.2019.11.24

Breast cancer represents one of the leading cancer diagnoses in women. Given the epidemiologic salience of the disease, a large body of research has been dedicated towards optimizing care and improving outcomes. One of the preeminent studies in breast cancer occurred with the NSABP B-06 trial establishing equivalence between breast-conserving surgery (BCS) followed by radiotherapy and mastectomy (1). Since this landmark finding, BCS accounts for approximately $65 \%$ of all operations for breast cancer (2). An unavoidable consequence of BCS is that a subset of women will ultimately have positive margins and require reoperation. Reports in the literature on re-excision rates after BCS have been found to range from $20 \%$ to $30 \%$ in large database studies $(3,4)$. Re-excision rates vary dramatically in single-institution reports and several patient, tumor, treatment and facility factors have been associated with reoperation after BCS $(3,5,6)$.

Optimizing re-excision rates after BCS is critical on both the patient level and the systems level. Reoperation after BCS results in additional time away from work and added mental and physical stress to patients. Furthermore, it results in delays in adjuvant therapy. On a systems level, reoperations result in increased medical costs and global improvement in rates would translate to significant savings to the healthcare system. In a study by Yu et al., a reduction in re-excision rate of $5.6 \%$ in a single-institution cohort reduced costs by approximately $\$ 200,000$ (7). Despite variable rates of re-excision in the literature and the impact to both patients and institutions, there is no well-established benchmark for re-excision rates. Given the documented variability, reoperation rate has been posed as a potential quality metric for institutions (8).

In the study by Landercasper $e t$ al., the authors leveraged the National Cancer Database (NCDB) to identify characteristics associated with reoperations with the overarching aim of finding potential targets for initiatives to reduce overall rates of re-excision and to discuss the integrity of re-excision for risk-profiling. The overall reoperation rate found in the study was $16.1 \%$, consistent with published rates. Several factors in adjusted analysis were noted to be associated with reoperation, including patient factors such as younger age, tumor factors such as lobular histology, larger tumor size, positive nodal status and treatment factors such as neoadjuvant chemotherapy use (9). Similar findings have been noted in the literature in both single-institution and large database studies $(3,5)$.

The disparate re-excision rates found in the literature in single-institution studies suggest that there is variability between facilities. Perhaps the most striking finding from the results of Landercasper and colleagues is the significant inter-facility variation in reoperation rate. The authors noted that the association with facility ID and reoperation rates remained despite adjusting for patient, tumor and treatment characteristics as well as facility volume and case mix (9). In a multi-institution analysis of 4 institutions and 3 large health plans, McCahill et al. also found similar and significant surgeon and institutional variation in reexcision rates (3). As Landercasper et al. acknowledge, the use of an administrative database limits a thorough analysis of between- and within-institution factors that could be driving this variability. This includes but is not limited to assessment of technical factors, margin assessment and 
judgment at the surgeon level, and method of localization at the institution level.

Given the relatively low complication profile and predominantly outpatient or short-stay practice associated with surgery for breast cancer, traditional metrics of quality are not applicable. The disparate rates in re-excision at the single-institution and national database level raise the question of whether underlying differences in care could be driving the variability and suggest that re-excision may be a plausible marker for quality assessment. Landercasper and colleagues stratified facilities into tiers of reoperation performance. After partial risk adjustment on the 5 highest factors associated with reoperation, $11 \%$ of facilities shifted performance quartiles compared with the unadjusted model. After adjusting for all 18 factors associated with reoperation, $21 \%$ of facilities shifted quartiles compared to the partial risk adjustment model. Overall, $25 \%$ of facilities shifted quartiles of performance in the fully adjusted model compared to the unadjusted model (9). These findings suggest that using unadjusted reoperation rates as a quality metric has high potential for bias. Furthermore, adjusted reoperation rates are highly sensitive to the parameters considered and should be used with caution.

The recent release of the Society of Surgical Oncology and American Society for Radiation Oncology (SSO-ASTRO) consensus guidelines in 2014 regarding surgical management of invasive breast cancer established that no tumor touching the inked margin of the surgical specimen represents a negative margin and completion of surgical therapy (10). Since the consensus statement, data indicate that re-excision rates have improved (4). The 2004-2015 time-period of the study by Landercasper $e t a l$. includes data from before and after the SSO-ASTRO consensus statement published in 2014. The authors performed a time-trend analysis to evaluate the impact of the SSO-ASTRO guidelines on reoperation rates. The authors found that reoperation rates from 2014-2015 were less than the expected value derived from modeling based on pre-guideline data. This finding translated to a statistically significant decline in rates after the introduction of the consensus statement. In a multiinstitution study by McCahill and colleagues investigating variability in re-excision after BCS prior to the publication of SSO-ASTRO consensus statement, the authors' findings highlighted the impact of a lack of clear guidelines on reexcision rates. In this study, nearly half of patients with close margins underwent re-excision and significant surgeonlevel variance in decisions regarding re-excision for negative margins was noted (3). Taken in concert, these findings suggest that the introduction of clear guidelines for margin status may address much of the variability in re-excision patterns and alter the lense with which we must view these findings given that the majority of data points occurred prior to the introduction of the SSO-ASTRO consensus statement. Evaluation of inter-facility variability after the SSO-ASTRO consensus guidelines is an important consideration for further study.

While the inter-facility variability is high, there is also consistent redemonstrations of patient and tumor factors associated with re-excision, such as young age, positive margins and lobular histology $(3,5,6,9)$. Several interventions and novel technologies have been associated with reduced reexcision rates. Interventions such as cavity shave margins have demonstrated success in decreasing reoperation rate, though shave margins often lead to higher tissue volume excision and can potentially result in impairment to cosmetic outcomes $(11,12)$. Alternate interventions such as intraoperative frozen section have also been associated with lower re-excision rates (13). Application of this technique is resource heavy for institutions and prolongs operative time. Consideration of developing a risk prediction model to identify patients at high risk for re-excision and to introduce targeted application of existing technologies may hold promise as an approach to reducing reoperation.

Though subject to the acknowledged limitations of largescale database analysis, the study by Landercasper and colleagues is the first to quantify effect size of contributors to re-excision and evaluate adjusted risk-profiling of institutions. The authors' findings demonstrate the need to establish a benchmark for re-excision rates and further investigate surgeon and institutional variation, particularly after the institution of SSO-ASTRO consensus guidelines. Reoperation rates are a potentially ripe area of quality improvement in the field of breast cancer surgery. Re-excisions are inevitable, but we can no longer accept wide variation in those rates as simply chance occurrence. Surgeons must lead the charge in defining this quality metric — or it will be defined for us by entities less engaged and knowledgeable of the nuances we face with every breast conservation attempt.

\section{Acknowledgments}

None.

\section{Footnote}

Conflicts of Interest: The authors have no conflicts of interest 
to declare.

Ethical Statement: The authors are accountable for all aspects of the work in ensuring that questions related to the accuracy or integrity of any part of the work are appropriately investigated and resolved.

\section{References}

1. Fisher B, Anderson S, Bryant J, et al. Twenty-year followup of a randomized trial comparing total mastectomy, lumpectomy, and lumpectomy plus irradiation for the treatment of invasive breast cancer. $\mathrm{N}$ Engl J Med 2002;347:1233-41.

2. Kummerow KL, Du L, Penson DF, et al. Nationwide trends in mastectomy for early-stage breast cancer. JAMA Surg 2015;150:9-16.

3. McCahill LE, Single RM, Aiello Bowles EJ, et al. Variability in reexcision following breast conservation surgery. JAMA 2012;307:467-75.

4. Havel L, Naik H, Ramirez L, et al. Impact of the SSOASTRO margin guideline on rates of re-excision after lumpectomy for breast cancer: a meta-analysis. Ann Surg Oncol 2019;26:1238-44.

5. Waljee JF, Hu ES, Newman LA, et al. Predictors of reexcision among women undergoing breast-conserving surgery for cancer. Ann Surg Oncol 2008;15:1297-303.

6. Wilke LG, Czechura T, Wang C, et al. Repeat surgery after breast conservation for the treatment of stage 0 to II breast carcinoma: a report from the National Cancer Data Base, 2004-2010. JAMA Surg 2014;149:1296-305.

Cite this article as: Elmore LC, Margenthaler JA. A tale of two operations: re-excision as a quality measure. Gland Surg 2019;8(6):593-595. doi: 10.21037/gs.2019.11.24
7. Yu J, Elmore LC, Cyr AE, et al. Cost analysis of a surgical consensus guideline in breast-conserving surgery. J Am Coll Surg 2017;225:294-301.

8. McCahill LE, Privette A, James T, et al. Quality measures for breast cancer surgery: initial validation of feasibility and assessment of variation among surgeons. Arch Surg 2009;144:455-62; discussion 462-3.

9. Landercasper J, Bennie B, Ahmad HF, et al. Opportunities to reduce reoperations and to improve inter-facility profiling after initial breast-conserving surgery for cancer. A report from the NCDB. Eur J Surg Oncol 2019;45:2026-36.

10. Moran MS, Schnitt SJ, Giuliano AE, et al. Society of Surgical Oncology-American Society for Radiation Oncology consensus guideline on margins for breastconserving surgery with whole-breast irradiation in stages I and II invasive breast cancer. Ann Surg Oncol 2014;21:704-16.

11. Wang K, Ren Y, He J. Cavity Shaving plus lumpectomy versus lumpectomy alone for patients with breast cancer undergoing breast-conserving surgery: a systematic review and meta-analysis. PLoS One 2017;12:e168705.

12. Chagpar AB, Killelea BK, Tsangaris TN, et al. A randomized, controlled trial of cavity shave margins in breast cancer. N Engl J Med 2015;373:503-10.

13. Boughey JC, Hieken TJ, Jakub JW, et al. Impact of analysis of frozen-section margin on reoperation rates in women undergoing lumpectomy for breast cancer: evaluation of the National Surgical Quality Improvement Program data. Surgery 2014;156:190-7. 